\title{
DEVELOPMENT OF ENTERPRISE PORTAL FOR INTELLIGENT MOLD SHOP
}

\author{
B.K. Choi, H.Y. Lee, D.W. Lee, and T.D. Kim \\ VMS Lab, Department of Industrial Engineering, Korea Advanced Institute of Science and \\ Technology (KAIST), 373-1, Guseong-dong, Yuseong-gu, Daejeon, Republic of Korea; \\ Email: bkchoi@vmslab.kaist.ac.kr
}

\begin{abstract}
Presented in the paper is an enterprise portal for intelligent mold shop (IMS) called IMS-portal to support the IMS system proposed earlier by the authors that consists of six software modules enabling to transform a skill-based moldshop into an intelligence-based one. The proposed IMS-portal is an enterprise $B P M$ portal in which the BPM (business process management) functionalities are embedded in the existing enterprise portal framework.
\end{abstract}

Key words: Intelligent mold shop; Enterprise portal; BPM (business process management) system; Enterprise BPM portal; IMS-portal.

\section{INTRODUCTION}

Mold shops are faced with a number of difficulties including severe global competition, increasing labor cost, increasing demand for lead-time reduction, and shortage of skilled manpower. Recently, a framework of IMS (intelligent mold shop) software system was proposed to help cope with these difficulties ${ }^{1}$. The proposed IMS system, which aims to substitute operators' skills and cognitive abilities by software modules and hardware components as much as possible so that the mold-machining shop can be run by fewer and less skilled operators, consists of six functional modules as depicted in Figure 1: NC-data filtering module for high-performance and error-free machining; Tooling plan module for robust and optimized toolselection; Setup plan module for standardized and robust setup; Virtual inspection module for machining quality assurance; Real-time monitoring module for unattended NC machining; Loading simulation module for optimized job-progress scheduling.

Please use the following format when citing this chapter:

Choi, B.K., Lee, D.W., Kim, T.D., 2006, in International Federation for Information Processing (IFIP), Volume 207, Knowledge Enterprise: Intelligent Strategies In Product Design, Manufacturing, and Management, eds. K. Wang, Kovacs G., Wozny M., Fang M., (Boston: Springer), pp. 498-504. 


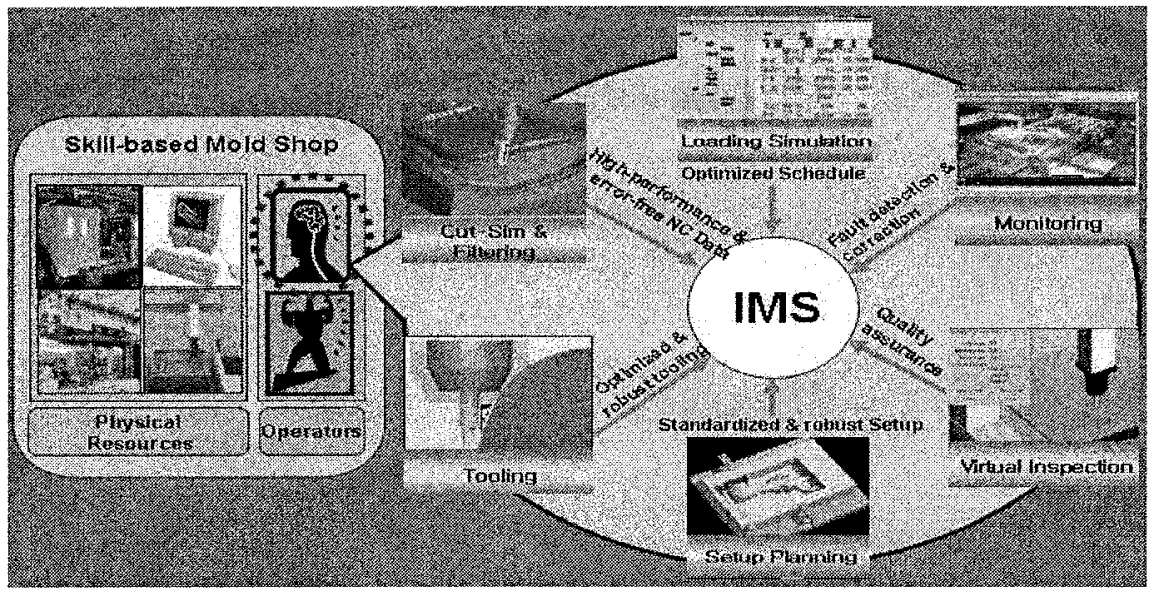

Figure 1. IMS (Intelligent Mold Shop) Framework ${ }^{1}$

A difficulty with the introduction of the IMS system is that it adds complexity in managing the use of diverse types of software systems and other resources. It is also required that the activities related to the IMS software system need to be integrated with other business processes and decision-making activities. Thus, we need a higher level information system framework that can provide a single-point gateway to all stakeholders while orchestrating diverse business processes involving the six IMS modules as well as other software systems in the mold shop.

\section{CONCEPTS OF ENTERPRISE PORTAL}

A search engine whose main goal is to facilitate access to information contained in documents spread throughout the internet has evolved to the concept of enterprise portal by integrating other functions such as virtual communities and by adding the ability to personalize interfaces and to access specialized contents ${ }^{2}$. As depicted in Figure 2, the search engine has evolved into portals along two paths: 1) it has evolved into a knowledge management system (KMS) and then to EKP (enterprise knowledge portal) for production, acquisition, transmission and management of knowledge focused on enterprise business processes ${ }^{3} ; 2$ ) it has evolved into EDMS and DW (data warehouse) and then into EIP (enterprise information portal) enabling a company to provide users with a single, personalized gateway to the information they need to make informed business decisions ${ }^{4}$. 


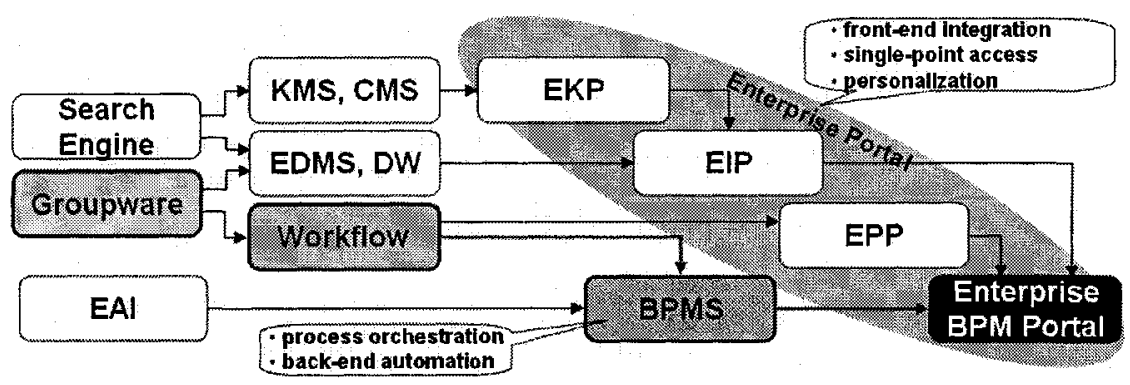

Figure 2. Enterprise Portal Concepts

Another line of development started from the concept of groupware that had evolved into workflow management systems (WfMS). A WfMS that provides intelligent user interfaces for workflow operations and augments with relevant process information is called an $\mathrm{EPP}^{5}$ (enterprise process portal). $\boldsymbol{B P} \boldsymbol{M}$ (business process management) system is the evolution of WfMS as well as of EAI (enterprise application integration) ${ }^{6}$; it is a software system that is to orchestrate operational business processes that interact with people and systems within and across organizations and are automatically driven by explicit process designs ${ }^{7,8}$.

Key words of BPMS are process orchestration and flexible automation of back-end processes, while existing enterprise portals focus on single-point access, front-end integration and personalized interfaces. By incorporating the functionalities of BPMS into the exiting framework of enterprise portal, we propose a new type of enterprise portal called Enterprise BPM Portal.

\section{IMS PORTAL}

In this paper, an enterprise BPM portal for a mold shop to orchestrate diverse business processes involving the six IMS modules as well as other software systems in the mold shop is called IMS-portal. An abstract framework of IMS-portal is depicted in Figure 3. The main functionalities of the IMS-portal are 1) orchestration of process-oriented services such as technical data processing and job progress management, 2) real-time support for decision-making services such as CTP (capable-to-promise) and outsourcing decision, 3 ) process-oriented management of information resources such as mold design data and manufacturing instruction data, and 4) proactive management of physical resources such as running NC machines and die-spotting machines. On top of these, it has to provide a single-point gateway via portlet ${ }^{9}$ that can be personalized for each of the stakeholders. 


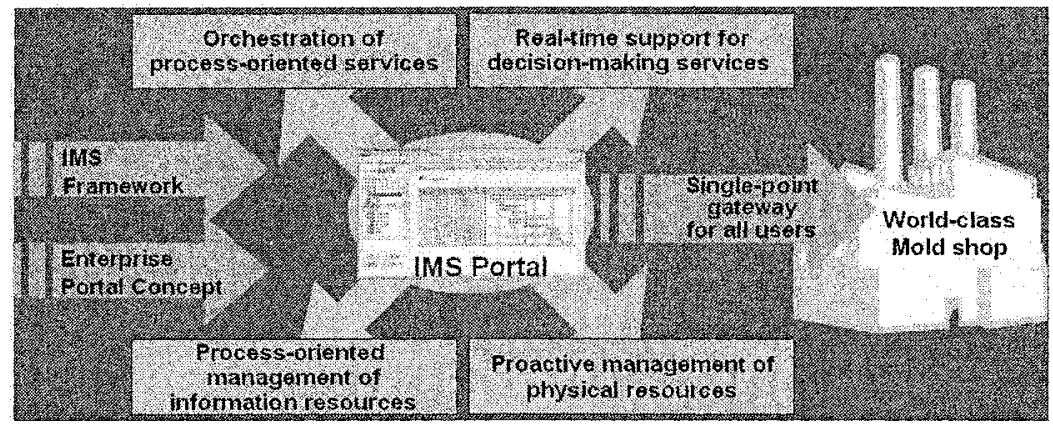

Figure 3. IMS-portal Framework

Shown in Figure 4 is a system architecture for the IMS-portal framework of Figure 3. The IMS-portal architecture consists of four layers: 1) resource layer containing all databases and business process repository, 2) application layer with all application software systems including the six IMS modules of Figure 1 (enclosed in the dashed rectangle), 3) orchestration layer providing milestone management and deliverable management functions and various process enactment services, 4) portal-service layer for providing searching and notification services to the stakeholders via the personalized portlet.

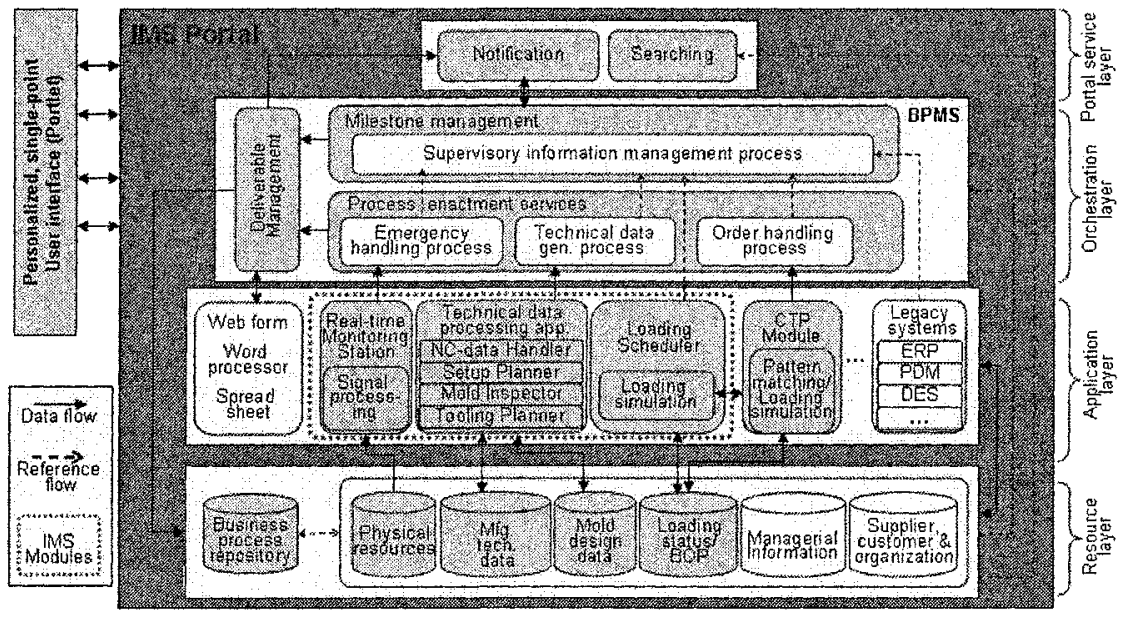

Figure 4. IMS-portal Architecture

Comparing Figure 4 with Figure 3, the deliverable management module is in charge of 'process-oriented management of information resources', and the emergency handling process and real-time monitoring station modules are in charge of 'proactive management of physical resources'. The order 
handling process module supporting the CTP Module is an example of 'realtime support for decision-making services'. There are two types of 'processoriented service': technical data-processing and job-progress management. The technical data-generation process module supporting NC-data Handler etc. is in charge of technical data processing services, and Loading Scheduler is in charge of (one type of) job-progress management services.

\section{IMPLEMENTATION OF IMS PORTAL}

The six IMS modules shown in Figure 1 had been implemented for a mold shop in Korea, and some of the modules have been used in the mold shop since 2004. On top of the IMS modules, an ad hoc prototype of the IMS-portal was implemented as depicted in Figure 5. Major modules of the ad hoc IMS-portal include Work-list handler, RTM (real-time monitoring) interface, Milestone planner for the loading schedule, and NC-data handler interface.

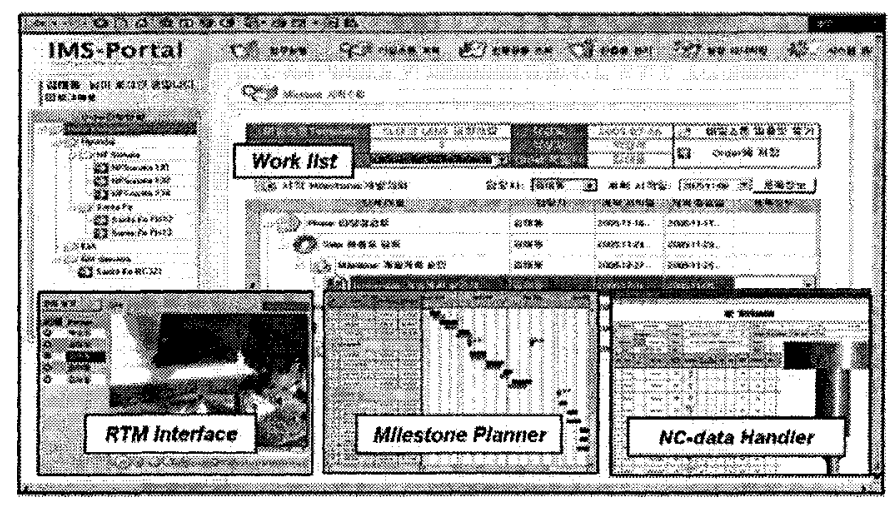

Figure 5. An Ad Hoc IMS-portal

A formal IMS-portal reflecting the architecture given in Figure 4 is under development. The modules in the orchestration layer are realized using an in-house BPM-engine named Harmony $\mathbb{B}$. The IMS-portal is a Web-based system employing the concept of portlet, and there are five main menus as shown in Figure 6:

1. Work-list menu is used to activate the 'Progress-oriented services worklist portlet' which provides the user with a list of pending workitems related to the Process enactment services (emergency handling, tech. data-generation and order handling) in the Orchestration layer of Figure 4. 
2. Mold-shop management menu is for the 'Job-progress monitoring portlet' which provides the user with a customized view of Milestone management in the Orchestration layer. It also controls the 'Physical resource monitoring portlet' for monitoring the current state of $\mathrm{NC}$ machines.

3. Information management menu is for the 'Information resources management portlet' which provides the user with a customized view of the Deliverables management in the Orchestration layer of Figure 4.

4. Application execution menu allows the user to directly invoke the various application software modules in the Application layer of Figure 4.

5. My information menu is for the 'Groupware portlet' which prides the user with personalized information services including notifying messages, e-mails, personal schedules, and portlet-configuration information.

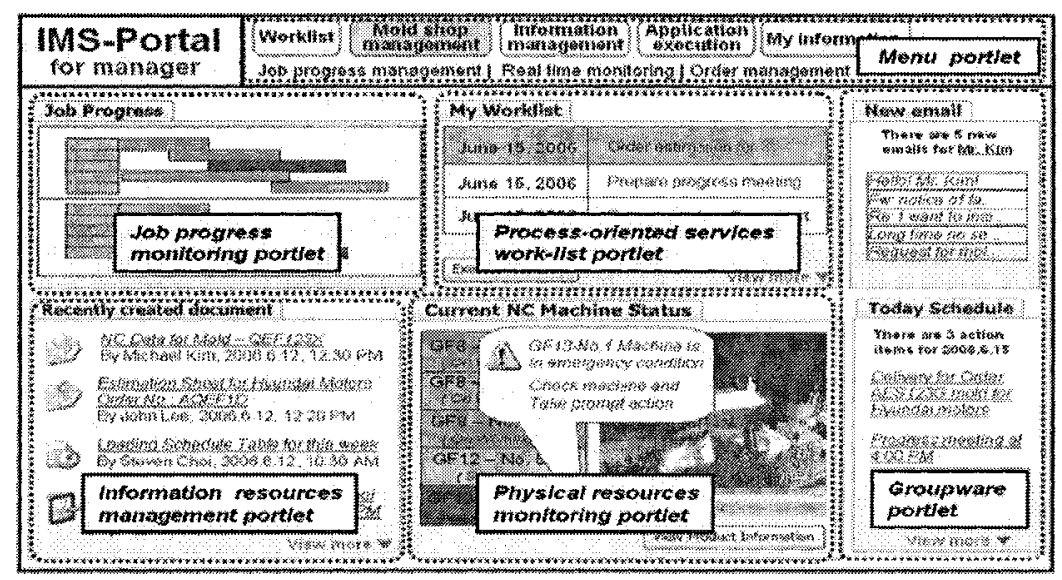

Figure 6. User Interface Design for IMS-portal

\section{CONCLUSION}

In this paper, a new type of enterprise portal called Enterprise BPM Portal is proposed and it is applied to an intelligent mold shop (IMS) to come up with a framework of IMS-portal. Software architecture of the IMSportal is also proposed that can provide a single-point gateway to all users while orchestrating diverse business processes involving various types of application software systems in the mold shop. An ad hoc IMS-portal was developed, and it is being upgraded to a 'formal' portal conforming to the proposed IMS-portal architecture. If fully developed and deployed, the proposed IMS-portal is expected to provide an unprecedented 'IT leverage' to mold-making companies. 


\section{REFERENCE}

1. B. K. Choi, K. Ko and B. H. Kim, Development of intelligent mold shop, Computer-Aided Design \& Applications, 2005, Vol. 2, No. 5, pp. 619-626

2. Claudia Dias, Corporate portals: a literature review of a new concept, International Journal of Information Management 21 (2001) 269-287

3. Firestone, J. M., DKMS Brief No. Eight: Enterprise information portals and enterprise knowledge portals, April 2000. [http://www.dkms.com/EKPandEIP.html].

4. Aneja, A., Rowan, C. \& Brooksby, B., Corporate Portal Framework for Transforming Content Chaos on Intranets, Intel Technology Journal, 2000, Q1, pp. 1-7.

5. Chi-Tsai Yang, Bin-Shiang Liang, Shung-Bin Yan, Feng-Jian Wang., An Intelligent and Personalized Enterprise Process Portal, Workflow Handbook 2004, pp207-220

6. Howard Smith and Peter Fingar: BPtrends, January 2004. [http://www.bpmi.org]

7. What does business process management mean for the IT industry?, KMworld Magazine, 2002, Sempember, Vol 11, Issue8, Ovum

8. BPM 2003 Market Milestone Report, Delphi, 2003

9. Sun Microsystems, Introduction to JSR 168-The Portlet Specification, http://developers.sun.com/prodtech/prtalserver/reference/techart/jsr168 